AN. MED INTERNA (Madrid) Vol. 19, N. $^{\circ} 7$, pp. 341-351, 2002

\title{
Tipos evolutivos de hipertensión portal prehepática en la rata
}

\author{
$\mathrm{M}^{\mathrm{a}}$ A. ALLER REYERO, B. DIÉGUEZ FERNÁNDEZ, M ${ }^{\mathrm{a}}$ P. NAVA HIDALGO*, \\ P. CUESTA ÁLVARO**, M. SÁNCHEZ GARCÍA***, H. J. DURÁN GIMÉNEZ-RICO, \\ M. A. LLAMAS MATÍAS, J. ARIAS PÉREZ \\ Cátedra de Cirugía. Facultad de Medicina. *Departamento de Fisiología Animal II. \\ Facultad de Ciencias Biológicas. **Centro de Proceso de Datos. ***Departamento de \\ Estadística e Investigación Operativa. Universidad Complutense. Madrid
}

\author{
EVOLUTIVE TYPES OF PREHEPATIC PORTAL HYPERTENSION OF \\ THE RAT
}

\begin{abstract}
RESUMEN
Objetivo: Estudio a largo plazo del modelo de hipertensión portal prehepática en la rata por triple ligadura estenosante portal.

Método: En los diferentes estadios evolutivos de la hipertensión portal (1 y1/2, 6, 12 y 14 meses) se realizó un análisis cluster de los animales que permite identificar tres grupos homogéneos (A, B y C) según el peso hepático.

Resultados: Los animales con atrofia hepática (grupo C) presentan disminución del peso corporal, esplénico y testicular, así como mayor desarrollo de circulación colateral portosistémica respecto de los grupos A y B.

Conclusión: Durante la evolución de la hipertensión portal disminuye de forma progresiva el porcentaje de animales perteneciente al grupo C y aumenta el correspondiente al grupo A, con hepatomegalia, en tanto que el grupo B permanece invariable.
\end{abstract}

PALABRAS CLAVE: Hipertensión portal. Rata. Hígado.

\section{ABSTRACT}

Objective: A long-term study of the prehepatic portal hypertension model by triple stenosing ligation of the portal vein in the rat.

Method: A cluster analysis of the animals in the different evolutive stages of portal hypertension (1 1/2, 6, 12 and 14 months) has been carried out. This analysis has made it possible to identify three homoge neous groups ( $A, B$ and $C$ ) in relationship to the liver weight.

Results: The animals with liver atrophy (group C) show a decrease of body, splenic and testicular weights as well as a higher development of the portosystemic collateral circulation in relation to the $A$ and $B$ groups.

Conclusion: The percentage of animals belonging to the group $C$ decrease progressively during the evolution of portal hypertension and those animals corresponding to the group $A$, in which is superior the liver weight, increase, while the B group does not change.

KEY WORDS: Portal hypertension. Rat. Liver.

Aller Reyero MA, Dieguez Fernández B, Nava Hidalgo MP, Cuesta Álvaro P, Sánchez García M, Durán Giménez-Rico HJ, Llamas Matías MA, Arias Pérez. J. Tipos evolutivos de hipertensión portal prehepática en la rata. An Med Interna (Madrid) 2002; 19: 341 -351.

\section{INTRODUCCIÓN}

El estudio de los mecanismos fisiopatológicos implicados en la hipertensión portal justifica la creación de nuevos modelos experimentales o el perfeccionamiento de los modelos existentes.

El modelo experimental más frecuentemente utilizado, para el estudio de la hipertensión prehepática, es el conseguido por ligadura estenosante simple de la vena porta en la rata $(1,2)$. En estadios evolutivos precoces, ésto es entre 28 y 45 días de la intervención, este modelo goza de gran predicamento. Dicho periodo evolutivo se caracteriza por la existencia de circulación portal colateral y un estado circulatorio hiperdinámico con hiperemia esplácnica $(3,4)$. Sin embargo a los 6 meses de evolución se ha descrito que desaparece la circulación hiperdinámica esplácnica $\mathrm{y}$, por lo tanto, la hipertensión portal se mantiene por otros factores, entre los cuales destaca la resistencia que ofrecen al flujo venoso esplácnico las colaterales portosistémicas. $(5,6)$.

La triple ligadura estenosante portal (TLEP) es una modificación de la ligadura estenosante simple que, al aumentar la resistencia inicial al flujo sanguíneo portal, agrava la evolución y, por esta razón, se ha propuesto como una alternativa válida para el estudio de la hipertensión portal prehepática experimental, tanto a corto como a largo plazo (7). Ya que los diferentes mecanismos que contribuyen al desarrollo de la hipertensión prehepática experimental permiten atribuir a esta patología diferentes fases evolutivas $(3,5)$, sería de mayor

Trabajo realizado con una Ayuda de la Universidad Complutense de Madrid. Ref. .nº PR52/00-8898.

Trabajo aceptado: 3 de abril de 2002

Correspondencia: M. A. Aller. Cátedra de Cirugía. Facultad de Medicina. Pza. Ramón y Cajal s/n. Universidad Complutense. 28040 Madrid. e-mail: maaller@ med.ucm.es. 
interés el estudio de las fases evolutivas tardías puesto que los mecanismos implicados en su producción, así como las alteraciones que se instauran, tendrían mayor semejanza con las que han sido descritas en la clínica humana, en tanto que son secundarias a la cronicidad de la hipertensión portal, entre otros factores (8). Así en el presente trabajo se ha estudiado la evolución a corto (45 días) y largo (6, 12 y 14 meses) plazo del modelo de hipertensión portal en la rata por TLEP, mediante la determinación de los tipos de circulación colateral, portohepática y portosistémica, que se desarrolla en éstos diferentes estadios evolutivos.

\section{MATERIAL Y MÉTODO}

\section{ANIMALES}

Se han utilizado 119 ratas macho de la cepa Wistar cuyos peso corporales oscilaron entre $230 \mathrm{~g}$ y $250 \mathrm{~g}$ procedentes del Animalario de la Universidad Complutense de Madrid. En todos los experimentos realizados para este estudio se han seguido la Normas de utilización y cuidado de los animales utilizados en la investigación y otros fines científicos, acordados por la Unión Europea en 1986 y publicadas en España en el Real Decreto 223/1988.

\section{DISEÑO EXPERIMENTAL}

Los animales fueron divididos para su estudio en Series control (RC) y con TLEP en cada uno de los estadios evolutivos estudiados: 45 días ( $\mathrm{RC} 45 \mathrm{~d} ; \mathrm{n}=18$; TLEP $45 \mathrm{~d}$; $\mathrm{n}=$ 27); 6 meses (RC $6 \mathrm{~m} ; \mathrm{n}=8$; TLEP $6 \mathrm{~m} ; \mathrm{n}=8$; TLEP $6 \mathrm{~m}$; $\mathrm{n}$ = 18), 12 meses (RC $12 \mathrm{~m} ; \mathrm{n}=14$; TLEP $12 \mathrm{~m} ; \mathrm{n}=17)$ y 14 meses (RC 14 m; $n=6$; TLEP $14 \mathrm{~m} ; \mathrm{n}=11$ ).

Todos los animales fueron sacrificados por exceso de éter y se determinaron los pesos corporal (PC), hepático (PH), esplénico (PE) y testicular (PTT).

\section{TÉCNICA DE LA TRIPLE LIGADURA ESTENOSANTE PORTAL (TLEP)}

Las ratas se anestesian con clorhidrato de ketamina $(75,5$ $\mathrm{mg} / \mathrm{kg}$ ) y diazepam $(5 \mathrm{mg} / \mathrm{kg})$ i.m. Se realiza una laparatomía media y las asas intestinales se desplazan a la izquierda del animal. A continuación se diseca la vena porta y se efectúan tres ligaduras estenosantes equidistantes en sus porciones superior, media e inferior (7). Las estenosis se calibran por ligadura simultánea (seda 4/0) alrededor de la vena porta y de una aguja $20 \mathrm{G}$ que se retira cuando finaliza el anudado, lo cual permite la reexpansión parcial de la vena porta (1).

\section{MÉTODO DE ESTUDIO DE LA CIRCULACIÓN COLATERAL}

Post-inducción anestésica se efectúa una laparatomía media y se estudian la áreas en las que se desarrolla la circulación venosa esplácnica colateral porto-hepática (hilio hepático: portoportal y vena hepática accesoria) y portosistémica (esplenorrenal: colateral esplenorrenal superior e inferior; gastroesofágica: colaterales paraesofágicas; colorrectal: colateral hemorroidal) (7). Según el diámetro de los vasos colate- rales se establecieron dos grados: grado 1 , si el diámetro es igual o inferior a $0,7 \mathrm{~mm}$ y grado 2 , si el diámetro es superior a $0,7 \mathrm{~mm}$.

\section{ESTUDIO DE LA VASCULOPATÍA VENOSA MESENTÉRICA (VVM)}

Se ha denominado vasculopatía venosa a la dilatación y tortuosidad de las ramas de la vena mesentérica superior. Se han considerado tres grados de vasculopatía venosa mesentérica: Grado 0: con aspecto macroscópico normal de las ramas de la vena mesentérica superior; Grado I: con dilatación y tortuosidad de las mencionadas ramas secundaria a la maniobra de Pringle; y Grado II: con dilatación y tortuosidad espontáneas de las ramas de la vena mesentérica superior.

\section{ANÁLISIS ESTADÍSTICO}

Los resultados se expresan como media $(\overline{\mathrm{X}}) \pm$ la desviación estándar (DE).

Para comparar las variables $\mathrm{PH}$, peso corporal inicial (PCI), incremento del peso corporal (IPC), PE y PTT entre las Series de animales control y las Series de animales con TLEP en cada periodo evolutivo se realizó la prueba de la t de Student para datos independientes. La comparación de éstas variables entre los distintos grupos A, B y C se realizó mediante ANOVA y un test de Tukey para contrastes a pos teriori. Para la comparación de la frecuencia de los distintos tipos de circulación colateral en los diferentes grupos A, B y $\mathrm{C}$ se empleó una prueba de $\chi^{2}$. Las diferencias se consideran estadísticamente significativas si $\mathrm{p}<0,05$.

Se ha realizado un Análisis Cluster con el objeto de obtener grupos homogéneos de ratas respecto al peso hepático en cada periodo evolutivo estudiado. Para ello se ha utilizado una técnica jerárquica de agregación y se ha determinado el número de clases observando el dendograma y los índices de agregación en cada paso.

\section{RESULTADOS}

\section{PESO HEPÁTICO}

Respecto de los animales control los grupos con TLEP presentan disminución $(p<0,001)$, del peso hepático a los 45 días de evolución, aumento $(\mathrm{p}<0,01)$ a los 6 y 12 meses en tanto que a los 14 meses del postoperatorio las diferencias no son estadísticamente significativas (Tablas I, II, III, IV).

En el grupo TLEP-45 d las diferencias entre los pesos hepáticos de los grupos A, B y C son estadísticamente significativas $(p<0,05)$ (Tabla I). Similares diferencias significativas $(p<0,05)$ se han hallado entre los pesos hepáticos de los grupos $\mathrm{A}, \mathrm{B}$ y $\mathrm{C}$ pertenecientes a los grupos TLEP-6 m, (Tabla II), TLEP-12 m (Tabla III) y TLEP-4 m (Tabla IV).

El análisis Cluster realizado en los animales con TLEP permite identificar grupos homogéneos de ratas los cuales han sido denominados $\mathrm{A}, \mathrm{B}$ y $\mathrm{C}$ según la disminución progresiva del peso hepático respectivamente.

En las Series TLEP-45 d y TLEP-6 m el grupo más numeroso corresponde a los animales con menor peso hepático o grupo $\mathrm{C}$ (TLEP-45 d grupo $\mathrm{C}, \mathrm{n}=15 ; 55,5 \%$ ); (TLEP-6 m 


\section{TABLA I}

PESO HEPÁTICO (PH), PESO CO RPO RAL INICIAL (PCI), INCREM ENTO DE PESO CO RPO RAL (IPC), PESO ESPLÉNICO (PE)

Y PESO TESTICULAR (PTT) EN RATAS CONTROL (RC) Y EN RATAS CON TRIPLE LIGADURA ESTENOSANTE DE LA VENA PORTA (TLEP) A LOS 45 DÍAS DE EVO LUCIÓN. TRAS REALIZAR UN ANÁLISIS CLUSTER DE LO S ANIM ALES Q UE CO M PO NEN

EL GRUPO DE TLEP, SEGÚN SU DISTRIBUCIÓN DEL PESO HEPÁTICO, ÉSTOS SE DIVIDIERON EN SUBGRUPOS A (TLEP-A), $B(T L E P-B)$ Y C (TLEP-C)

\begin{tabular}{|c|c|c|c|c|c|}
\hline Serie & $\begin{array}{l}\mathrm{PH} \\
(\mathrm{g})\end{array}$ & $\begin{array}{l}\mathrm{PCl} \\
(\mathrm{g})\end{array}$ & $\begin{array}{r}\text { IPC } \\
(g)\end{array}$ & $\begin{array}{l}\text { PE } \\
(g)\end{array}$ & $\begin{array}{l}\text { PTT } \\
(\mathrm{g})\end{array}$ \\
\hline $\begin{array}{c}R C \\
(n=18)\end{array}$ & $17,52 \pm 2,87$ & $242,71 \pm 6,41$ & $208,72 \pm 42,30$ & $0,74 \pm 0,09$ & $3,24 \pm 0,41$ \\
\hline $\begin{array}{c}\text { TLEP } \\
(n=27)\end{array}$ & $13,91 \pm 3,47$ & $241,89 \pm 7,12$ & $187,85 \pm 53,52$ & $1,11 \pm 0,29$ & $3,02 \pm 0,44$ \\
\hline $\begin{array}{c}\text { TLEP-A } \\
(n=3)\end{array}$ & $20,20 \pm 2,86^{*}$ & $249 \pm 4,91$ & $254,8 \pm 22,44^{*}$ & $1,28 \pm 0,34$ & $3,48 \pm 0,21 * *$ \\
\hline $\begin{array}{l}\text { TLEP-B } \\
(\mathrm{n}=9)\end{array}$ & $15,99 \pm 0,67 *$ & $240,18 \pm 10,43$ & $222,24 \pm 22,13^{*}$ & $1,20 \pm 0,41$ & $2,85 \pm 0,50$ \\
\hline $\begin{array}{l}\text { TLEP-C } \\
(n=15)\end{array}$ & $11,40 \pm 1,69^{\circ}$ & $241,49 \pm 3,97$ & $153,83 \pm 44,80$ & $1,03 \pm 0,14$ & $3,03 \pm 0,39$ \\
\hline
\end{tabular}

$\mathrm{X} \pm \mathrm{DE}$

$\mathrm{p}<0,001$ valor ES del grupo TLEP respecto de $\mathrm{RC} ; \quad * \mathrm{p}<0,05 ; * * p<0,01$ valor ES respecto TLEP-C; $\quad$ - $p<0,05 ;$ valor ES respecto TLEP-B

TABLA II

PESO HEPÁTICO (PH), PESO CORPORAL INICIAL (PCI), INCREM ENTO DE PESO CORPORAL (IPC), PESO ESPLÉNICO (PE) Y PESO TESTICULAR (PTT) EN RATAS CONTROL (RC) Y EN RATAS CON TRIPLE LIGADURA ESTENO SANTE DE LA VENA PORTA (TLEP) A LOS 6 M ESES DE EVO LUCIÓ N. TRAS REALIZAR UN ANÁLISIS CLUSTER DE LOS ANIM ALES Q UE CO M PO NEN EL GRUPO DE TLEP, SEGÚN SU DISTRIBUCIÓ N DEL PESO HEPÁTICO, ÉSTOS SE DIVIDIERON EN SUBGRUPOSA (TLEP-A), $B(T L E P-B)$ Y C (TLEP-C)

\begin{tabular}{|c|c|c|c|c|c|}
\hline Grupo & $\begin{array}{l}\mathrm{PH} \\
(\mathrm{g})\end{array}$ & $\begin{array}{l}\mathrm{PCl} \\
(\mathrm{g})\end{array}$ & $\begin{array}{r}\text { IPC } \\
(\mathrm{g})\end{array}$ & $\begin{array}{l}\text { PE } \\
(g)\end{array}$ & $\begin{array}{l}\text { PTT } \\
(\mathrm{g})\end{array}$ \\
\hline $\begin{array}{c}\mathrm{RC} \\
(n=8)\end{array}$ & $11,63 \pm 0,64$ & $232,42 \pm 5,38$ & $259,80 \pm 22,61$ & $0,70 \pm 0,10$ & $3,64 \pm 0,25$ \\
\hline $\begin{array}{c}\text { TLEP } \\
(n=18)\end{array}$ & $15,38 \pm 3,05$ & $237,72 \pm 11,30$ & $337,78 \pm 95,63$ & $1,23 \pm 0,30$ & $3,59 \pm 0,28$ \\
\hline $\begin{array}{l}\text { TLEP-A } \\
(n=2)\end{array}$ & $21,25 \pm 1,62 *^{\bullet}$ & $247,90 \pm 10,89$ & $452,2 \pm 78,91^{*}$ & $1,54 \pm 0,13$ & $3,91 \pm 0,11$ \\
\hline $\begin{array}{l}\text { TLEP-B } \\
(n=6)\end{array}$ & $17,07 \pm 0,62^{*}$ & $242,60 \pm 6,92$ & $408,73 \pm 61,25^{*}$ & $1,24 \pm 0,26$ & $3,57 \pm 0,15$ \\
\hline $\begin{array}{l}\text { TLEP-C } \\
(n=10)\end{array}$ & $13,19 \pm 1,46^{\bullet}$ & $232,76 \pm 11,62$ & $272,32 \pm 58,92$ & $1,16 \pm 0,33$ & $3,53 \pm 0,32$ \\
\hline
\end{tabular}

$\bar{X} \pm \mathrm{DE}$

$p<0,05 ; \quad p<0,01 ; \quad p<0,001$ valor ES del grupo TLEP respecto de RC

$* p<0,05$; valor ES respecto TLEP-C; $\quad \bullet p<0,05$; valor ES respecto TLEP-B

grupo $\mathrm{C}, \mathrm{n}=55,5 \%$ ) (Tabla V, Figs. 1 y 2). En la Serie TLEP-12 $\mathrm{m}$ el mayor número de animales corresponde al grupo B (TLEP-12 m grupo B; $\mathrm{n}=7 ; 41,1 \%$ ) (Tabla V, Fig. 3). A su vez en la Serie TLEP-14 m el mayor porcentaje de animales corresponde al grupo A (TLEP-14 $\mathrm{m} ; \mathrm{n}=6 ; 54,5 \%)$ (Tabla V, Fig. 4).

Por el contrario, el grupo menos numeroso en las Series TLEP-45 d y TLEP- $6 \mathrm{~m}$ es el grupo A (TLEP-45 d grupo $\mathrm{A}: \mathrm{n}=3 ; 11,1 \%$, TLEP-6 $\mathrm{m}$ grupo $\mathrm{A}, \mathrm{n}=2 ; 11,1 \%$ ) (Tabla $\mathrm{V}$, Figs. 1 y 2) en tanto que en las Series TLEP-12 m y TLEP-14 $\mathrm{m}$ el menor porcentaje de animales corresponde al grupo $\mathrm{C}$
(TLEP-12 $\mathrm{m}$ grupo $\mathrm{C}, \mathrm{n}=4 ; 23,5 \%$; TLEP-14 $\mathrm{m}$ grupo $\mathrm{C}, \mathrm{n}=$ $1 ; 9,09 \%$ ) (Tabla V, Figs. 1 y 2).

Según estos resultados en las ratas con TLEP la disminución del peso hepático (grupos C) es más frecuente en los dos primeros estadios evolutivos estudiados, esto es, a los 45 días y 6 meses del postoperatorio. Asimismo en estadíos evolutivos posteriores (12 y 14 meses) aumenta el porcentaje de animales que presentan mayor peso hepático (grupos A) (Tabla V). Estas diferencias en la distribución de los animales en los distintos grupos (A, B y C) según el periodo evolutivo considerado son estadísticamente significativas $(p=0,02)($ Tabla V). 


\section{TABLA III}

PESO HEPÁTICO (PH), PESO CORPORAL INICIAL (PCI), INCREM ENTO DE PESO CO RPO RAL (IPC), PESO ESPLÉNICO (PE) Y PESO TESTICULAR (PTT) EN RATAS CONTROL (RC) Y EN RATAS CON TRIPLE LIGADURA ESTENOSANTE DE LA VENA PORTA (TLEP) AL AÑO DE EVO LUCIÓN. TRAS REALIZAR UN ANÁLISIS CLUSTER DE LOS ANIM ALES Q UE CO M PO NEN EL GRUPO DE TLEP, SEGÚN SU DISTRIBUCIÓN DEL PESO HEPÁTICO, ÉSTO S SE DIVIDIERO N EN SUBGRUPOS A (TLEP-A), $B(T L E P-B)$ Y C (TLEP-C)

\begin{tabular}{|c|c|c|c|c|c|}
\hline Serie & $\begin{array}{l}\mathrm{PH} \\
\text { (g) }\end{array}$ & $\begin{array}{l}\mathrm{PCl} \\
(\mathrm{g})\end{array}$ & $\begin{array}{l}\text { IPC } \\
(g)\end{array}$ & $\begin{array}{l}\text { PE } \\
\text { (g) }\end{array}$ & $\begin{array}{l}\text { PTT } \\
(g)\end{array}$ \\
\hline $\begin{array}{c}R C \\
(n=8)\end{array}$ & $14,91 \pm 2,11$ & $255,80 \pm 16,04$ & $438,96 \pm 144,09$ & $0,92 \pm 0,10$ & $3,98 \pm 0,39$ \\
\hline $\begin{array}{l}\text { TLEP } \\
(n=18)\end{array}$ & $19,16 \pm 4,41$ & $241,26 \pm 11,75$ & $460,58 \pm 127,83$ & $1,39 \pm 0,46$ & $3,59 \pm 0,61$ \\
\hline $\begin{array}{l}\text { TLEP-A } \\
(n=2)\end{array}$ & $24,36 \pm 0,66^{*} \bullet$ & $243,17 \pm 10,45$ & $575,83 \pm 57,82^{*}$ & $1,61 \pm 0,71$ & $3,99 \pm 0,40$ \\
\hline $\begin{array}{l}\text { TLEP-B } \\
(n=6)\end{array}$ & $17,92 \pm 0,79 *$ & $243,77 \pm 11,93$ & $464,11 \pm 55,70^{*}$ & $1,29 \pm 0,23$ & $3,55 \pm 0,58$ \\
\hline $\begin{array}{l}\text { TLEP-C } \\
(n=10)\end{array}$ & $13,53 \pm 1,36^{\bullet}$ & $234,00 \pm 13,32$ & $281,50 \pm 77,55^{\bullet}$ & $1,25 \pm 0,23$ & $3,06 \pm 0,56$ \\
\hline
\end{tabular}

$\mathrm{X} \pm \mathrm{DE}$

$p<0,01$ valor ES del grupo TLEP respecto de RC; $\quad * p<0,05$; valor ES respecto TLEP-C $\quad \cdot p<0,05$; valor ES respecto TLEP-B

\section{TABLA IV}

PESO HEPÁTICO (PH), PESO CORPORAL INICIAL (PCI), INCREM ENTO DE PESO CORPO RAL (IPC), PESO ESPLÉNICO (PE) Y PESO TESTICULAR (PTT) EN RATAS CONTROL (RC) Y EN RATAS CON TRIPLE LIGADURA ESTENO SANTE DE LA VENA PORTA (TLEP) A LOS 14 M ESES DE EVO LUCIÓN. TRAS REALIZAR UN ANÁLISIS CLUSTER DE LOS ANIM ALES Q UE CO M PO NEN EL GRUPO DE TLEP, SEGÚN SU DISTRIBUCIÓN DEL PESO HEPÁTICO, ÉSTOS SE DIVIDIERON EN SUBGRUPOS A (TLEP-A), $B(T L E P-B)$ Y C (TLEP-C)

\begin{tabular}{cccccc}
\hline Grupo & $\begin{array}{c}\text { PH } \\
(\mathrm{g})\end{array}$ & $\begin{array}{c}\mathrm{PCl} \\
(\mathrm{g})\end{array}$ & $\begin{array}{c}\text { IPC } \\
(\mathrm{g})\end{array}$ & $\begin{array}{c}\text { PE } \\
(\mathrm{g})\end{array}$ & $\begin{array}{c}\text { PTT } \\
(\mathrm{g})\end{array}$ \\
\hline $\begin{array}{c}\mathrm{RC} \\
(\mathrm{n}=6)\end{array}$ & $14,26 \pm 1,77$ & $238,32 \pm 4,99$ & $257,75 \pm 34,11$ & $0,77 \pm 0,18$ & $3,99 \pm 0,21$ \\
$\begin{array}{c}\text { TLEP } \\
(\mathrm{n}=11)\end{array}$ & $14,69 \pm 2,40$ & $227,60 \pm 4,65$ & $344,04 \pm 45,12$ & $1,09 \pm 0,34$ & $3,54 \pm 0,64$ \\
$\begin{array}{c}\text { TLEP-A } \\
(\mathrm{n}=6)\end{array}$ & $16,37 \pm 0,96^{*}$ & $226,57 \pm 5,41$ & $365,93 \pm 21,57^{*}$ & $1,29 \pm 0,35$ & $3,60 \pm 0,85$ \\
$\begin{array}{c}\text { TLEP-B } \\
(\mathrm{n}=4)\end{array}$ & $13,46 \pm 1,01^{*}$ & $229,25 \pm 4,19$ & $340,50 \pm 21,32^{*}$ & $0,84 \pm 0,13$ & $3,59 \pm 0,09$ \\
$\begin{array}{c}\text { TLEP-C } \\
(n=1)\end{array}$ & 9,51 & 277,20 & 226,80 & 0,91 & 2,88 \\
\end{tabular}

$\bar{x} \pm \mathrm{DE}$

$p<0,01$ valor ES del grupo TLEP respecto de $\mathrm{RC} ; \quad * p<0,05$; valor ES respecto TLEP-C

\section{PESOS CORPORALES, ESPLÉNICOS Y TESTICULARES}

El peso corporal de los animales con TLEP, es variable en los distintos estadios evolutivos estudiados respecto de los valores control. Así, en las Series TLEP-6 m y TLEP-14 m el aumento es ES ( $p<0,05$ y p< 0,01, respectivamente) en relación con las correspondientes Series control (Tablas II y IV).

En todas las Series TLEP estudiadas el menor peso corporal corresponde a los animales de los grupos $\mathrm{C}$, en tanto que el mayor incremento de peso corporal lo presentan los animales de los grupos A. El incremento del peso corporal en los grupos A y B de los animales con TLEP es estadísticamente significativo ( $\mathrm{p}<0,05)$, respecto de los grupos C. (Tablas I a IV). A su vez, el mayor incremento de peso corporal entre los animales con TLEP lo presentan los correspondientes a 14 meses de evolución (TLEP-14 m).

El incremento del peso esplénico, en las Series TLEP es estadísticamente significativo a los $6(\mathrm{p}<0,001)$ y 12 meses $(\mathrm{p}<0,01)$ respecto de las correspondientes Series control. El mayor peso esplénico lo presentan en todos los estadios evo- 
TABLA V

DISTRIBUCIÓ N DE LOS ANIM ALES CON TRIPLE LIGADURA ESTENOSANTE DE LA VENA PO RTA (TLEP) TRAS REALIZAR UN ANÁLISIS CLUSTER, SEGÚN SU PESO HEPÁTICO, EN GRUPOS A, B Y C. DE ACUERDO CON LA PRUEBA $\chi^{2}$ LA DIFERENCIA DE FRECUENCIA EN DISTINTOS GRUPOS EN LOS PERÍO DO S EVO LUTIVO S CO NSIDERADOS ES SIGNIFICATIVA $P=0,02$

\begin{tabular}{ccccccccc}
\hline $\begin{array}{c}\text { Grupo } \\
\mathrm{n}\end{array}$ & \multicolumn{2}{c}{ TLEP-45 $\mathrm{d}$} & \multicolumn{2}{c}{ TLEP-6 } & \multicolumn{3}{c}{ TLEP-12 m } & \multicolumn{2}{c}{ TLEP-14 m } \\
\hline A & 3 & 11,1 & 2 & 11,1 & 6 & 35,2 & 6 & 54,5 \\
B & 9 & 33,3 & 6 & 33,3 & 7 & 41,2 & 4 & 36,3 \\
C & 15 & 55,5 & 10 & 55,5 & 4 & 23,5 & 1 & 9,09 \\
\hline
\end{tabular}

lutivos los animales pertenecientes a los grupos A, y el menor peso esplénico en TLEP-45 d, TLEP-6 $\mathrm{m}$ y TLEP-12 m corresponde a los grupos C (Tablas I a IV).

Aunque no existen diferencias estadísticamente significativas entre el peso testicular de los animales con TLEP y los controles, cuando se consideran los primeros divididos en los grupos A, B y C, se observa que las ratas con TLEP de los grupos $\mathrm{C}$ presentan menor peso testicular respecto de los grupos A, si bien ésta diferencia sólo es estadísticamente significativa $(\mathrm{p}<0,01)$ a los 45 días de evolución (Tablas I a IV). Por lo tanto, en los animales con TLEP el menor peso hepático se corresponde con menor peso testicular.

\section{CIRCULACIÓN COLATERAL}

La circulación colateral portohepática constituida por las colaterales porto-portales y por la vena hepática accesoria, es en la mayoría de los estadios estudiados, más frecuente en los grupos B y $\mathrm{C}$ constituidos por los animales con TLEP que tienen menor peso hepático. El calibre reducido de las colaterales porto-portales se considera que es inapropiado para conseguir la revascularización portal del hígado a su través (8). Sin embargo, la vena hepática accesoria puede alcanzar gran calibre y se supone que revasculariza el hígado siguiéndo un trayecto entre los lóbulos lateral izquierdo y caudado (8). En los animales con TLEP se observa que el desarrollo de la vena hepática accesoria aumenta su frecuencia a lo largo de la evolución. Así a los 6, 12 y 14 meses es más frecuente que a los 45 días del postoperatorio. Asímismo, durante la evolución aumenta la frecuencia de esta colateral portohepática en los grupos A, B y C (Figs. 1 a 4) aunque destaca su elevada incidencia en los grupos C de TLEP-6m $(70 \%, n=7)$ y TLEP-12m $(100 \%, \mathrm{n}=4)$ asi como en los grupos A y B de TLEP14m (grupo A: 83,3\%, $n=5$; grupo B: 75\% $n=3$ ) (Figs. 1 a 4).

Respecto de la circulación colateral portosistémica, el tipo esplenorenal superior es más frecuente que el tipo esplenorenal inferior (ERi) en todos los estados evolutivos de las ratas con TLEP. A su vez la, circulación ERs se desarrolla con similar frecuencia en los tres grupos estudiados (A: $\mathrm{n}=10,90,9 \% ; \mathrm{B}: \mathrm{n}=$ $22,78,5 \% ; C: n=29 ; 87,8 \%$ ) en tanto que la circulación ERi de grado II es más frecuente en los grupos A $(n=5 ; 45,45 \% ; B, n=$ $11,39,28 \%)(\mathrm{p}=0,04)$ y $\mathrm{B}(\mathrm{n}=8 ; 47 \%)(\mathrm{p}=0,009)$ y la ERi de grado I en los grupos $C(n=15 ; 45,4 \% ; p=0,04)$ (Figs. 1 a 4$)$.

El tipo de circulación colateral paraesofágica (PE) se ha encontrado en proporción variable en todos los estadios evolutivos, siendo más frecuente en TLEP-6m y TLEP-12 m.
Considerando su frecuencia según los tres grupos estudiados en los diferentes periodos evolutivos, predomina su incidencia en el grupo A en TLEP-45 d; en TLEP-6 m es más frecuente en los grupos A y B y en TLEP-12 m la circulación PE alcanza máxima incidencia en los tres grupos (Figs. 1 a 4).

Por último la circulación colateral hemorroidal $(\mathrm{H})$ suele ser menos frecuente que la circulación colateral PE. Su desarrollo máximo (grado II) se alcanza con mayor frecuencia en estadios evolutivos tardíos de la hipertensión portal (TLEP$6 \mathrm{~m}$, TLEP-12 $\mathrm{m}$ y TLEP-14 $\mathrm{m}$ ) y predomina en los grupos $\mathrm{B}$ y C (B: $n=9 ; 32,1 \%, C: n=11 ; 33,3 \%)$ (Figs. 1 a 4 ).

\section{VASCULOPATÍA VENOSA MESENTÉRICA}

La incidencia de vasculopatía venosa mesentérica (VVM) es mayor a los 45 días post-TLEP que en estadios posteriores (6, 12 y 14 meses). En este estadio evolutivo precoz (TLEP$45 \mathrm{~d})$ la frecuencia de vasculopatía mesentérica, tanto de grado I como de grado II, es mayor $(\mathrm{p}=0,008)$ en los grupos B y $\mathrm{C}$ (VVM grado $\mathrm{I}=\mathrm{B}: \mathrm{n}=6 ; 21,43 \%, \mathrm{C}: \mathrm{n}=16 ; 48,5 \%$; VVM grado II = B: $n=8 ; 28,5 \%, C: n=9 ; 27,3 \%)$ (Figs. 1 a 4$)$. En periodos más tardíos de la evolución la mayor frecuencia de vasculopatía venosa mesentérica la presentan los grupos B y C. A los 6 meses, en el grupo C la VVM es de grado I en el $50 \%$ de los casos $(n=5)$ y de grado II en el $30 \%(n=3)$. Por último, a los 12 meses de evolución, en el grupo A ningun animal presenta VVM, en tanto en el grupo C, es de grado I en el $75 \%(n=3)$ y de grado II en el $25 \%$ de los animales $(n=1)$.

\section{DISCUSIÓN}

El estudio evolutivo realizado demuestra la validez de la TLEP para obtener un modelo de hipertensión portal prehepática a largo plazo en la rata. Sin embargo, los resultados de este trabajo muestran que algunas de las alteraciones que son propias del estadío evolutivo precoz (TLEP-45d) varían en frecuencia e intensidad durante los estadíos tardíos (TLEP-6 $\mathrm{m}$, TLEP-12 $\mathrm{m}$ y TLEP-14 $\mathrm{m}$ ).

Para el estudio de las variaciones evolutivas que presenta este modelo experimental se ha distribuido a los animales según su peso hepático en tres grupos denominados A, B y C mediante un análisis Cluster. Esta clasificación de los animales con hipertensión portal se fundamenta en un trabajo previo realizado con ratas a los 45 días de TLEP en que, debido a la amplia oscilación 

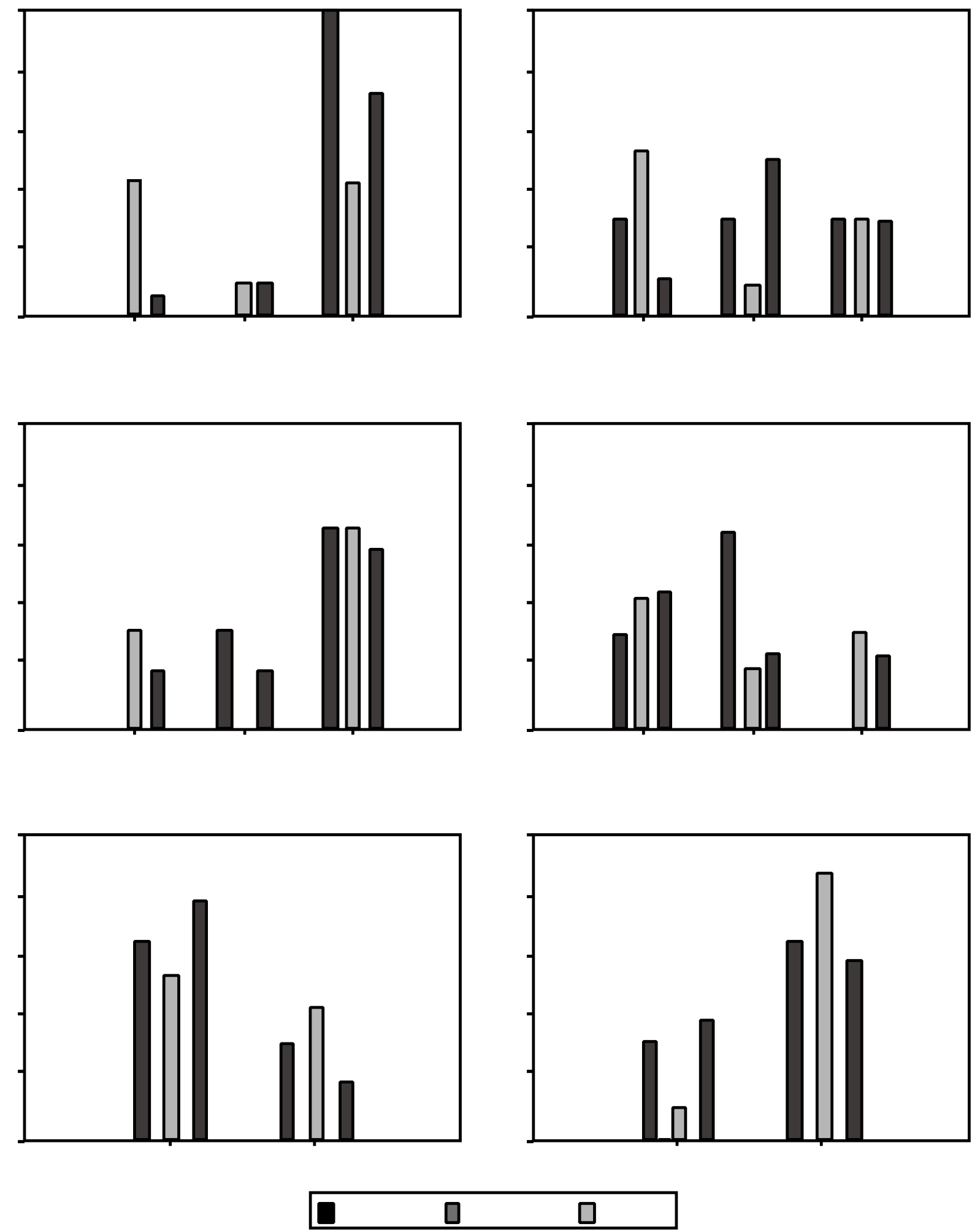

Fig. 1. Incidencia y grados de circulación colateral portosistémica: esplenorenal superior (ERS), esplenorenal inferior (ERI), paraesofágica $(\mathrm{PE})$, hemorroidal $(\mathrm{H})$ y porto-hepática: a través de la vena hepática accesoria (VHA) y portoportal (PP) en los grupos $A, B$ y C de ratas con hipertensión portal por triple ligadura estenosante de la vena porta (TLEP) a los 45 días de evolución postoperatoría. 

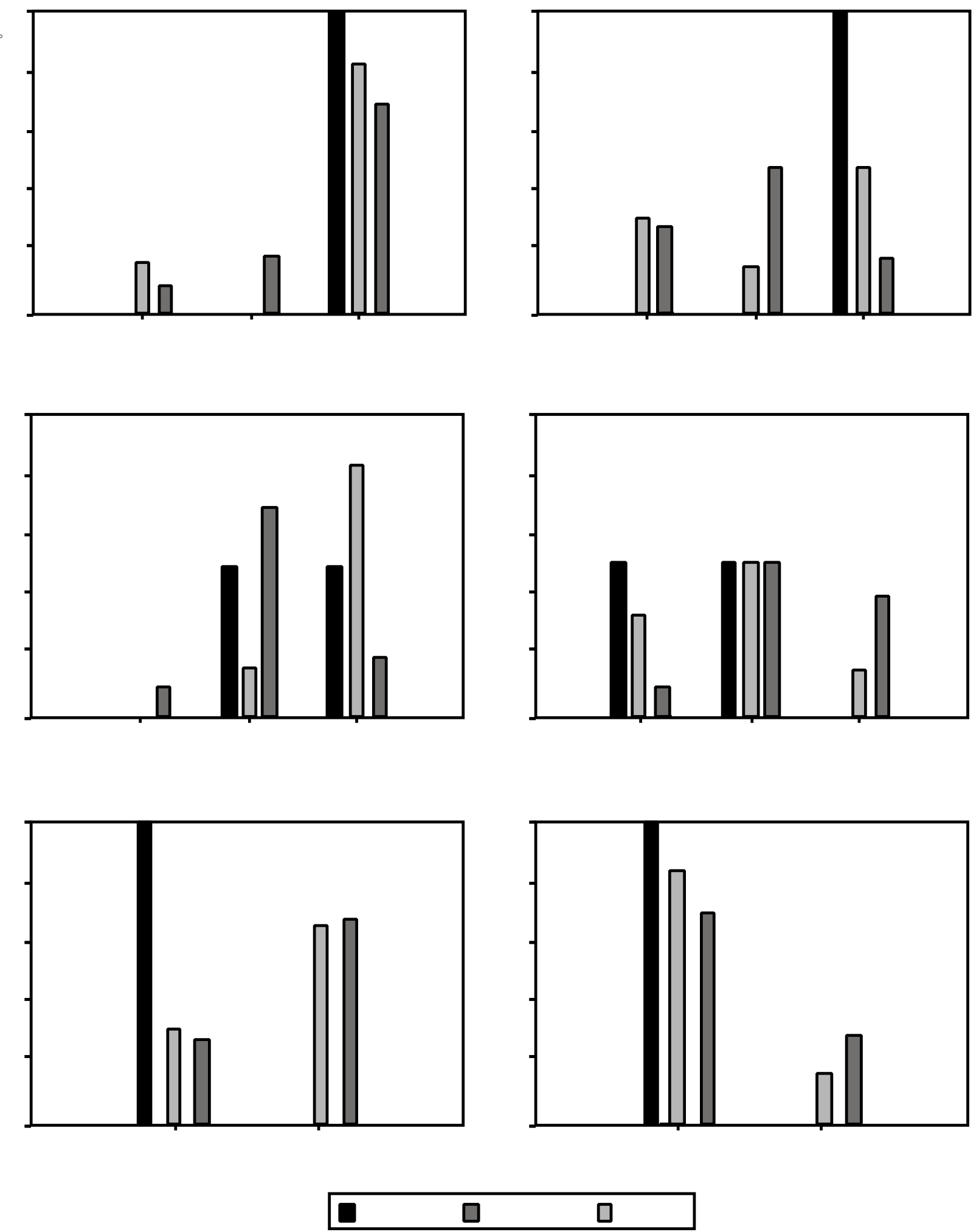

Fig. 2. Incidencia y grados de circulación colateral portosistémica: esplenorenal superior (ERS), esplenorenal inferior (ERI), paraesofágica $(\mathrm{PE})$, hemorroidal $(\mathrm{H})$ y porto-hepática: a través de la vena hepática accesoria (VHA) y portoportal (PP) en los grupos $A, B$ y C de ratas con hipertensión portal por triple ligadura estenosante de la vena porta (TLEP) a los 6 meses de evolución postoperatoría. 

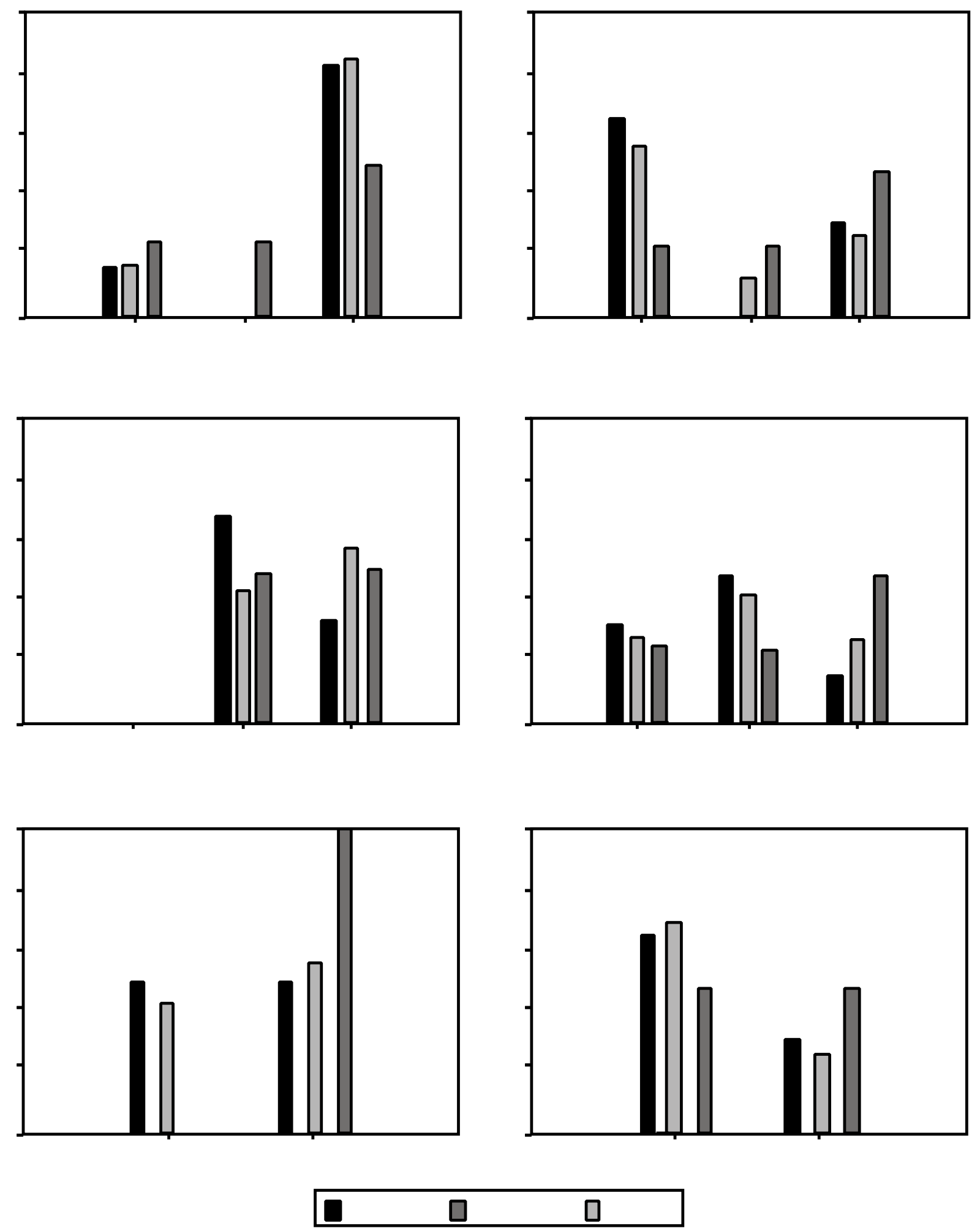

Fig. 3. Incidencia y grados de circulación colateral portosistémica: esplenorenal superior (ERS), esplenorenal inferior (ERI), paraesofágica $(\mathrm{PE})$, hemorroidal $(\mathrm{H})$ y porto-hepática: a través de la vena hepática accesoria (VHA) y portoportal (PP) en los grupos A, B y C de ratas con hipertensión portal por triple ligadura estenosante de la vena porta (TLEP) a los 12 meses de evolución postoperatoría. 

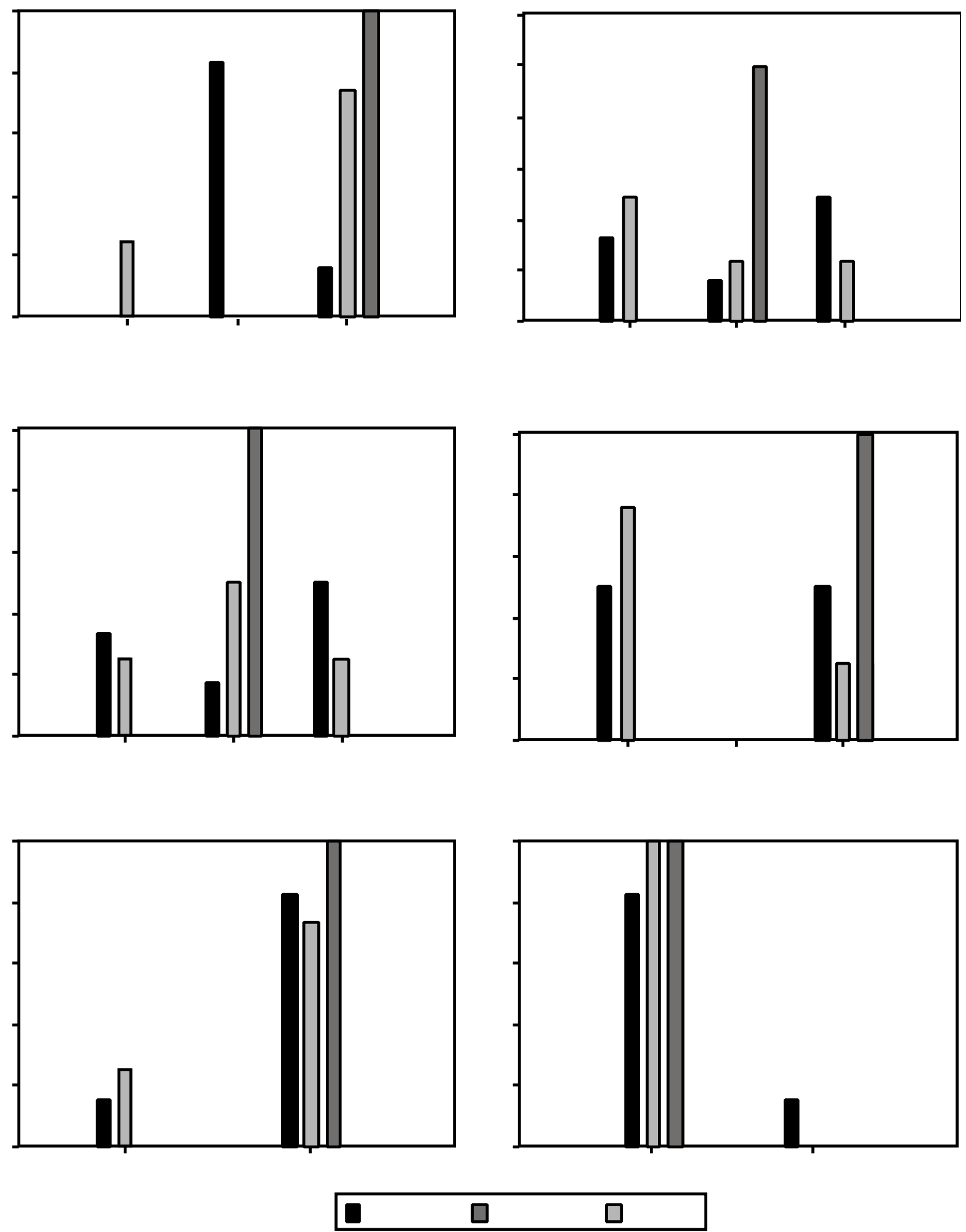

Fig. 4. Incidencia y grados de circulación colateral portosistémica: esplenorenal superior (ERS), esplenorenal inferior (ERI), paraesofágica $(\mathrm{PE})$, hemorroidal $(\mathrm{H})$ y porto-hepática: a través de la vena hepática accesoria (VHA) y portoportal (PP) en los grupos $\mathrm{A}, \mathrm{B}$ y C de ratas con hipertensión portal por triple ligadura estenosante de la vena porta (TLEP) a los 14 meses de evolución postoperatoría. 
del peso hepático, se optó para su estudio en una distribución en tres grupos según el peso del hìgado fuese superior (subgrupo A), igual (subgrupo B) o inferior (subgrupo C) al propio de los animales control (7). En el presente trabajo dicha distribución demuestra que el porcentaje de animales con mayor peso hepático (grupo A) aumenta progresivamente a lo largo de la evolución post-TLEP, en tanto que disminuye el porcentaje correspondiente al grupo $\mathrm{C}$ que está constituido por las ratas con menor peso hepático. Por lo tanto, la atrofia hepática, que caracteriza los estadíos evolutivos precoces de la hipertensión portal prehepática en la rata $(3,9)$ quizá sea una alteración reversible a largo plazo. Los grupos A, aunque caracterizados por el aumento del peso hepático, también presentan circulación colateral portosistémica y, por lo tanto, este tipo de evolución a corto y largo plazo no invalida el modelo experimental de hipertensión portal por triple ligadura estenosante en la rata.

En este modelo experimental de hipertensión portal prehepática se produce aumento del peso esplénico en todas las fases evolutivas (Tablas I a IV). Sin embargo, el mayor aumento del peso esplénico a lo largo de la evolución lo presentan los animales que tienen mayor peso hepático (grupos A). La asociación de hepatomegalia y esplenomegalia en ratas con TLEP podría sugerir que la interrelación hígado-bazo es diferente a la descrita en otros modelos experimentales de hipertensión portal. Por ejemplo, en ratas hepatectomizadas se ha demostrado que el bazo ejerce un efecto inhibidor en la regeneración hepática (10). Por esta razón, la esplenectomía ejerce una acción beneficiosa en ratas cirróticas, puesto que favorece la regeneración hepática, entre otros mecanismos porque preserva la función de las células de Kupffer y, en particular su secreción de TNF- $\alpha$ (11). Sin embargo, en ratas con estenosis portal de 6 meses de evolución se ha propuesto que el bazo tiene una función moduladora de la presión portal, yá que la ligadura de la arteria esplénica, pero no la esplenectomía, reduce la presión portal $(5,6)$. Este efecto protector del bazo en la hipertensión portal ha sido atribuido a la preservación de las colaterales esplenorenales que facilitan el drenaje de la sangre venosa esplácnica (5).

El aumento progresivo de la incidencia de hepatomegalia durante la evolución de éste modelo experimental se podría considerar un hallazgo de interés porque sugiere que es una característica propia de la hipertensión portal prehepática crónica en la rata. En un estudio previo también se ha demostrado que el $35 \%$ de las ratas con TLEP de un año de evolución presentan un incremento estadísticamente significativo del peso hepático respecto de los animales control (8).

Yá que la hepatomegalia se asocia con aumento del peso corporal, ambas alteraciones podrían tener una relación etiopatogénica. En éste sentido, se ha considerado que, si el aumento del peso corporal fuera causado por exceso de tejido adiposo, el aumento de peso hepático podría reflejar la existencia de un hígado graso en éstos animales con hipertensión portal prehepática crónica. Resultados aún no publicados, demuestran la existencia de degeneración grasa progresiva del hígado en las ratas con TLEP y, por lo tanto, permiten establecer una relación entre el incremento asociado de los pesos hepático y corporal, éste último por obesidad. Asímismo, la frecuente asociación de hepatomegalia con el desarrollo de circulación colateral porto-hepática a través de la vena hepática accesoria sugiere la importancia en su producción de la revascularización portal del hígado y, por lo tanto, de mediadores de procedencia intestinal (Figs. 3 y 4).

La disminución de peso testicular es otra alteración propia de los animales con TLEP perteneciéntes al grupo $\mathrm{C}$ y, por consiguiente, su incidencia es mayor en los períodos evolutivos precoces de la hipertensión portal. La atrofia testicular se ha correlacionado con el grado de shunt porto-sistémico yá que es mayor en ratas con anastomosis porto-cava que con hipertensión portal prehepática $(12,13)$. Asímismo, se ha sugerido que la reducción de la derivación portosistémica podría contribuir a la reversibilidad de la atrofia testicular en ratas cirróticas tratadas con factor de crecimiento similar a Insulina (IGF-1) (14). Estudios previos en ratas con TLEP45d yá demostraban que la atrofia testicular se asocia a menor peso hepático y mayor desarrollo de circulación colateral portosistémica (7). Los resultados del presente trabajo, en estadíos evolutivos más avanzados de la hipertensión portal, evidencian que el peso testicular de las ratas con hipertensión portal perteneciéntes a los grupos $\mathrm{B}$ y $\mathrm{C}$ es inferior al corrrespondiente del grupo A. Yá que las diferencias en el peso testicular entre éstos grupos de animales con hipertensión portal prehepática persisten en todos los períodos evolutivos estudiados, se podría considerar que sus variaciones se correlacionan con las que presenta el peso hepático y, por lo tanto, constituiría otra de las alteraciones que permitirían definir cada tipo evolutivo de éste modelo experimental (7).

La dilatación y tortuosidad de las ramas de la vena mesentérica superior es una alteración macroscópica frecuentemente relacionada con alteraciones vasculares microscópicas del duodeno, como son el aumento significativo del número y del diámetro de los vasos de la mucosa y submucosa (15). Se ha propuesto que éstas alteraciones histológicas podrían ser secundarias a la excesiva liberación de mediadores proinflamatorios por las abundantes células cebadas que infiltran la mucosa y la submucosa duodenales de las ratas con hipertensión portal prehepática precoz (15-17). En éste supuesto, el aumento de la prevalencia de vasculopatía venosa mesentérica en las ratas con hipertensión portal del grupo C, se podría atribuir a la mayor intensidad de la respuesta inflamatoria intestinal. Según ésta hipotética consideración, la disminución progresiva del porcentaje de animales del grupo C durante la evolución de la hipertensión portal experimental implicaría la remisión progresiva de la respuesta inflamatoria intestinal causada por la brusca instauración de la hipertensión venosa esplácnica que produce la TLEP.

Asímismo, en los estadíos evolutivos tardíos el aumento progresivo del porcentaje de animales perteneciéntes al grupo A, caracterizados por un mayor peso hepático y corporal, menor desarrollo de circulación colateral portosistémica y menor incidencia de vasculopatía venosa mesentérica, representaría la instauración gradual de las alteraciones propias de la hipertensión portal crónica en éste modelo experimental.

En conclusión, las diferentes alteraciones que presentan las ratas con TLEP permiten definir tres grupos de animales (A, B y C) que, a su vez, representan las características evolutivas predominantes en los estadíos precoces (grupo C) y tardíos (grupos B y A).

\section{AGRADECIMIENTOS}

Agradecemos su colaboración a la Dra. Dña. Pilar Bringas de la Lastra, Directora Técnica del Animalario de la UCM, así como a D. Oscar Grijota Chousa, D. Manuel Solís Martínez y D. Fernándo del Barrio Revilla, pertenecientes al personal técnico de dicho Animalario. Asímismo, queremos expresar nuestro agradecimiento a Amalia Pérez por la transcripción mecanográfica del manuscrito. 


\section{Bibliografía}

1. Chojkier M, Groszmann RJ. Measurement of portal-systemic shunting in the rat using $\gamma$-labeled microspheres. Am J Physiol 1981; 240: G 371- G5.

2. Bosch J, Pizcueta P, Feu F, Fernández M, García-Pagan JC. Pathophysiology of portal hypertension. Gastroenterol Clinics North Am 1992; 21: 1-14.

3. Sikuler E, Kravetz D, Groszmann RJ. Evolution of portal hypertension and mechanisms involved in its maintenance in a rat model. Am J Physiol 1985; 248: G 618-G25.

4. Lee F Y, Colombato LA, Albillos A, Groszmann RJ. Administration of $\mathrm{N}^{\mathrm{w}}$-Nitro-L-arginine ameliorates portal-hypertensive rats. Gastroenterology 1993; 105: 1464-70.

5. Lin PW, Shan YS. Effects of splenectomy and splenic artery ligation on the portal pressure in portal hypertensive rats. J Surg Res 1992; 53: 621-4.

6. Lin PW, Shan YS. Effects of splenectomy on portal pressure in short and long-term portal hypertensive rats. J Formosan Med Asso 1992; 91: 487-90.

7. Rodríguez-Fabián G, Monterde G, Dieguez B, Aller MA, Arias J. Hipertensión portal a largo plazo en la rata por triple ligadura estenosante de la vena porta. An Med Interna (Madrid) 2000; 17: 137-41.

8. Aller MA, Nava MP, Dieguez B, Palma MD, Arias JL, Arias J. Hipertensión portal prehepática crónica en la rata por triple ligadura estenosante portal. Cir Esp 2001; 69: 103-7.

9. Halvorsen JF, Myking AO. Prehepatic portal hypertension in the rat. Immediate and long-term effects on portal vein and long-term effects on portal vein stenosis, followed by occlusion of the portal vein and splenorenal collaterals. Eur Surg Res 1979; 11: 89- 98.

10. Suzuki S, Nakamura S, Serizawa S, Sakaguchi T, Konno H, Muro H, et al. Role of Kupffer cell and the spleen in modulation of endotoxin-induced liver injury after partial hepatectomy. Hepatology 1996; 24: 219-25.

11. Murata K, Shiraki K, Sugimoto K, Takase K, Nakano T, Furusaka A, et al. Splenectomy enhances liver regeneration through tumor necrosis factor (TNF)- $\alpha$ following dimethylnitrosamine-induced cirrhotic rat model. Hepato-Gastroenterol 2001; 48: 1022-7.

12. Van Thiel DH, Gavaler JS, Slone FL, Cobb CF, Smith WI, et al. Is feminization in alcoholic men due in part to portal hypertension: a rat model. Gastroenterology 1980; 78: 81-91.

13. Van Thiel DH, Gavaler JS, Cobb CF, McClain CJ. An evaluation of the respective roles of portosystemic shunting and portal hypertension in rats upon the production of gonadal dysfunction in cirrhosis. Gastroenterology $1983 ; 85: 154-9$

14. Castilla-Cortazar I, García M, Quiroga J, Díez N, Díez-Caballero F, Calvo A, et al. Insulin-like growth factor-I reverts testicular atrophy in rats with advanced cirrhosis. Hepatology 2000; 31: 592-600.

15. Díez-Arias JA, Aller MA, Palma MD, Arias JL, Muñíz E, Sánchez M, et al. Increased duodenal mucosa infiltration by mast cells in rats with portal hypertension. Dig Surg 2001: 18: 34-40.

16. Díez-Arias JA, Aller MA, Nava MP, Gragera R, Dieguez B, Muñíz E, et al. Portal hypertensive duodenopathy in the rat. Span J Surg Res 2001; 4: 25-9.

17. Monterde G, Rodríguez-Fabian G, Vara E, López L, Arias J, Aller MA, et al. Increased plasma levels of corticosterone and prolactin and decreased T3 and T4 levles in short-term prehepatic portal hypertension in rats. Dig Dis Sci 2000; 45: 1865-71. 